\title{
CARACTERIZACIÓN GENÉTICA Y MOLECULAR DE Pseudomonas Aeruginosa CAUSANTE DE INFECCIONES EN UCI DE TRES CIUDADES DE COLOMBIA.
}

\section{GENETIC AND MOLECULAR CHARACTERIZATION OF Pseudomonas Aeruginosa CAUSING INFECTIONS IN ICU OFTHREE CITIES IN COLOMBIA.}

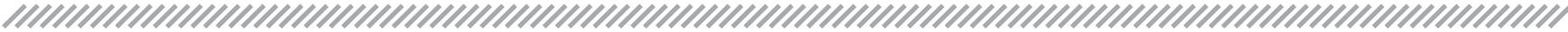

AUTORES: Abril Riaño Deisy Julieth ${ }^{1}$, Castro Cardozo Betsy², Moncada Guayazán María Victoria ${ }^{3}$, Corredor Rozo Zayda Lorena, Márquez Ortiz Ricaurte Alejandro ${ }^{5}$, Olarte Narda ${ }^{6}$, Valderrama Alberto ${ }^{7}$, Tovar Catalina ${ }^{8}$, Buelvas Francisco 9 , Moncayo Jairo ${ }^{10}$, Guaca Yina ${ }^{11}$, Reyes Niradiz ${ }^{12}$, Vanegas Gómez Natasha ${ }^{13}$, Escobar Pérez Javier $^{14}$
\end{abstract}

\section{RESUMEN}

Pseudomonas aeruginosa, bacteria ubicua que puede generar infecciones complicadas en pacientes hospitalarios, incrementando los índices de morbimortalidad debido al incremento de resistencia a los antimicrobianos de uso clínico, en especial los de última opción terapéutica como los carbapenémicos por la adquisición de determinantes de resistencia a través de elementos genéticos móviles principalmente.

OBJETIVO: Caracterizar el perfil de resistencia y características moleculares de P. aeruginosa, aislada de pacientes adultos con diagnóstico de infección en tres unidades de cuidados intensivos en Colombia.

MÉTODOS: Se analizaron pacientes con infecciones por P.aeruginosa en UCI adultos. A los aislamientos bacterianos se les determinó el perfil de susceptibilidad a 12 antibióticos y se amplificaron genes de resistencia a $\beta$-lactámicos, quinolonas, sulfonamidas y plataformas genéticas como integrón clase 1 y 2 . La relación genética por medio de PFGE y MLST.

RESULTADOS: En el estudio se analizaron 40 pacientes de los cuales 23(57,5\%) pertenecen a una UCI en la ciudad de Pereira. Las principales fuentes de aislamiento de los microorganismos son hemocultivos $13(32,5 \%)$ y urocultivos $11(27,5 \%)$. Los perfiles de resistencia SAM-FOX y SAM-FOX-SXT se presentaron en $6(15,0 \%)$ y $4(10,0 \%)$ aislamientos respectivamente. Las $\beta$-lactamasas más frecuentes fueron de tipo blaTEM, en $8(20,0 \%)$, blaSHV $7(17.5 \%)$ y blaCTX-M 3(7.5\%), carbapenemasas de tipo blaKPC-2 y blaVIM en $4(9.7 \%)$ y $3(7.5 \%)$. Los aislamientos presentan un comportamiento policlonal con 28 pulsotipos. Los aislamientos productores de KPC-2 y VIM se encuentran asociados al ST235 y ST111 respectivamente.

CONCLUSIONES: las infecciones generadas en las UCI de las entidades participantes presentan gran variabilidad y con una moderada resistencia a carbapenémicos asociados a la presencia de KPC-2 y VIM asociados al clon pandémico ST235 y ST111.

PALABRAS CLAVE: Bacteria, infecciones, aislamiento, pacientes, genética.

\begin{abstract}
Pseudomonas aeruginosa, ubiquitous bacteria that can generate complicated infections in hospital patients, increasing morbidity and mortality rates due to increased antimicrobial resistance in clinical use, especially the last therapeutic option as carbapenems for the acquisition of resistance determinants through mainly mobile genetic elements.
\end{abstract}

OBJECTIVE: To characterize the resistance profile and molecular characteristics of P. aeruginosa isolated from adult patients with a diagnosis of infection in three intensive care units in Colombia.

${ }^{1}$ Laboratorio de Genética Molecular Bacteriana- Universidad El Bosque Instituto de Biotecnología. Universidad Nacional de Colombia.

${ }^{2}$ Grupo de Vigilancia Epidemiología - Hospital el Tunal

${ }^{3}$ Grupo de Enfermedades Tropicales y Resistencia Bacteriana - Universidad el Sinu

${ }^{4}$ Centro de Biología Molecular y Biotecnología - Universidad Tecnológica de Pereira

${ }^{5}$ Genetica y Biología Molecular - Universidad de Cartagena

${ }^{6}$ Institute. University of Technology of Sydney, Australia 
METHODS: Patients with P. aeruginosa infections in adults were analyzed UCI. A bacterial isolates were determined profile of susceptibility to 12 antibiotics and resistance genes were amplified $\beta$-lactams, quinolones, sulfonamides and genetic platforms like integron class 1 and 2. The genetic relationship by PFGE and MLST.

RESULTS: In the study 40 patients of which 23 (57.5\%) analyzed belong to an ICU in the city of Pereira. The main sources of isolating microorganisms are 13 blood cultures (32.5\%) and urine-11 (27.5\%). Resistance profiles SAM-SAM-FOX and FOX-SXT occurred in $6(15.0 \%)$ and $4(10.0 \%)$ isolates respectively. The $\beta$-lactamases frequently were blaTEM type in 8 (20.0\%), blaSHV seven (17.5\%) and blaCTX-M 3 (7.5\%), carbapenemases blaVIM blaKPC-2 and type 4 $(9.7 \%)$ and $3(7.5 \%)$. Isolates presented a polyclonal behavior pulsotypes 28. The KPC-producing isolates 2 and VIM are associated with the ST235 and ST111 respectively.

CONCLUSION: those generated in ICUs participating entities infections are highly variable and moderate resistance to carbapenems associated with the presence of KPC-2 and VIM associated with the pandemic clone ST235 and ST111.

KEYWORDS: Bacteria, infections, isolation, patients, genetics.

\section{INTRODUCCIÓN}

P. aeruginosa es una bacteria ubicua y debido a su capacidad de sobrevivir en ambientes con mínimos nutrientes y tolerar condiciones ambientales extremas puede persistir en ambientes comunitarios y hospitalarios. En este último, puede ser aislado de equipos, antisépticos y superficies en general. P. aeruginosa hace parte de la flora normal humana en menos del 5\% de las personas sanas, sin embargo, este porcentaje aumenta hasta el $50 \%$ en pacientes hospitalizados.

Las infecciones asociadas a aislamientos multirresistentes de P. aeruginosa aumentan la morbilidad, mortalidad, estancias hospitalarias y los costos de tratamiento. Este patógeno puede desarrollar resistencia a los antimicrobianos de uso clínico por medio de dos mecanismos principales, la adquisición de genes de resistencia través de elementos genéticos móviles (plásmidos, transposones e integrones) y por mutaciones de su genoma que alteran la regulación de proteínas constitutivas.

P.aeruginosa presenta altos frecuencia de resistencia a beta-lactámicos, aminoglucósidos y fluoroquinolonas, en especial en los causantes de infecciones en pacientes en UCI. El principal mecanismo de resistencia a betalactámicos es la adquisición de ß-lactamasas que pueden ser de espectro ampliado como PSE, CARB y TEM o de espectro extendido tanto de clase A y D, principalmente. En el primer grupo se han detectado variantes de las familias TEM, SHV, CTX-M, PER, VEB, GES e IBC y en el segundo grupo enzimas tipo OXA. Adicionalmente pueden sobre-expresar cefalosporinasa tipo AmpC, generando resistencia a todos los antibióticos de esta clase hasta cefalosporinas de 4ta generación (cefepime), pero no a carbapenémicos. Como ocurre en Enterobacterias, la prevalencia de diferentes clases de carbapenemasas en P. aeruginosa se ha incrementado en los últimos años. Cuatro familias de metalo-ß-lactamasas han sido identificadas en aislamientos de P. aeruginosa, IMP, VIM, SPM y GIM (con sus respectivas variantes). Recientemente se han identificado aislamientos de esta bacteria productores de B-lactamasas de clase A, del tipo KPC y OXA, principalmente OXA-40.El clon predominante de este género pertenece a ST175 (VIM-2 MDR), esta cepa surgió en Hospital Universitario español en al años 2008 asociado a aislamientos multirresistentes. También Australia China y Australia se reportó el clon ST277 de desde 2006, sin embargo este ST ha sido registrado en varias partes del mundo, mostrando un gran potencial de virulencia y diseminación internacional. En Colombia, se ha demostrado la presencia de ST308, ST111 y ST235 siendo este último ampliamente reconocido como responsables de los brotes en toda Europa, Asia y América del Sur.

\section{MATERIALES Y MÉTODOS}

Se realizó un estudio observacional prospectivo en el cual se analizaron 40 pacientes con infecciones por Pseudomonas aeruginosa en UCI adultos pertenecientes a tres instituciones de Colombia durante entre noviembre de 2014 y Julio de 2015. Todos los pacientes cumplieron los criterios de inclusión del estudio, como ser mayor de 18 años, tener diagnóstico de infección de acuerdo con los criterios de CDC, no presentar infecciones polimicrobianas y firmar el consentimiento informado.

En la caracterización se confirmó género y especie por la amplificación de gen especie específico, determinación 
del perfil de susceptibilidad a doce antibióticos de uso clínico (PTZ, SAM, FOX, CAZ, MER, IMP, DOR, STX, CIP, GEN, AMK, COL) por medio del método de dilución en agar siguiendo las recomendaciones del CLSI 2015. En la caracterización molecular se amplificaron genes de resistencia a $\beta$-lactámicos (blaTEM, blaSHV y blaCTX-M, blaKPC, blaVIM, blaOXA48, blaGES, blaNDM, blaIMP y ampC plasmídico), aminoglucósidos (aac6'ib), quinolonas (qnr, mcbg y aac6'ib-cr), sulfomanidas (sul1, sul2, sul3) e integrón Clase 1 y clase 2 . La diferenciación de blaKPC-2 y blaKPC-3 se realizó por medio de restricción enzimática con RsaI, estas variantes fueron confirmadas por secuenciación. La relación genética de los aislamientos se realizó por medio de PFGE (Electroforesis en Gel de Campo Pulsado) y MLST.

\section{RESULTADOS}

Origen de los aislamientos

En el estudio se analizaron 40 pacientes adultos con diagnóstico de infección adquirida en la UCI, de los cuales $23(57,5 \%)$ pertenecen a una institución hospitalaria en la ciudad de Pereira, 12(30,0\%) a Montería y $6(15,0 \%)$ a Bogotá. De los cuales $25(62,5 \%)$ son hombres con un promedio de edad de 46 años. Por cada paciente incluido el estudio se analizó únicamente un aislamiento bacteriano recuperado de las siguientes fuentes: hemocultivos 13(32,5\%), urocultivo $11(27,5 \%)$, Secreciones bronquiales $8(20,0 \%)$, liquido peritoneal $7(17,5 \%)$ y puntas de catéter $2(5,0 \%)$.

Distribución del diagnóstico de infección

De los 40 pacientes incluidos al estudio, 15(37,5\%) provenían de pacientes con diagnóstico de septicemia, $7(17,5 \%)$ de neumonía, $5(12,5 \%)$ de infección de vías urinarias y peritonitis, $1(2,5 \%)$ de presentan bronquitis sin signos de neumonía, anemia post-hemorrágica aguda y enfermedad pulmonar obstructiva crónica respectivamente, a $6(15,0 \%)$ pacientes no fue posible determinar el diagnóstico.

\section{Perfil de resistencia}

De los 40 aislamientos analizados presentaron resistencia 26(65,0\%) a STX, 17(42,5\%) a FOX, CRO, SAM, y MER, 11(27,5\%) a PTZ, 10(25,0\%) FEP y CAZ, $8(20,0 \%)$ CIP, $7(17.5 \%)$ a GEN y IMP, $6(15,0 \%)$ a AK, $5(12,5 \%)$ a DOR y ningún aislamiento fue resistente a COL. (Figura 1). El perfil de resistencia STX y MERSTX se presentó en $8(20,0 \%)$ y $7(17.5 \%)$ aislamientos respectivamente, el perfil de multirresistencia GEN, AK, CAZ, MER, CIP, STX, PTZ se identificó en $4(10,0 \%)$ aislamientos. Sólo dos aislamientos fueron susceptibles a todos los antibióticos evaluados. (Figura 2).

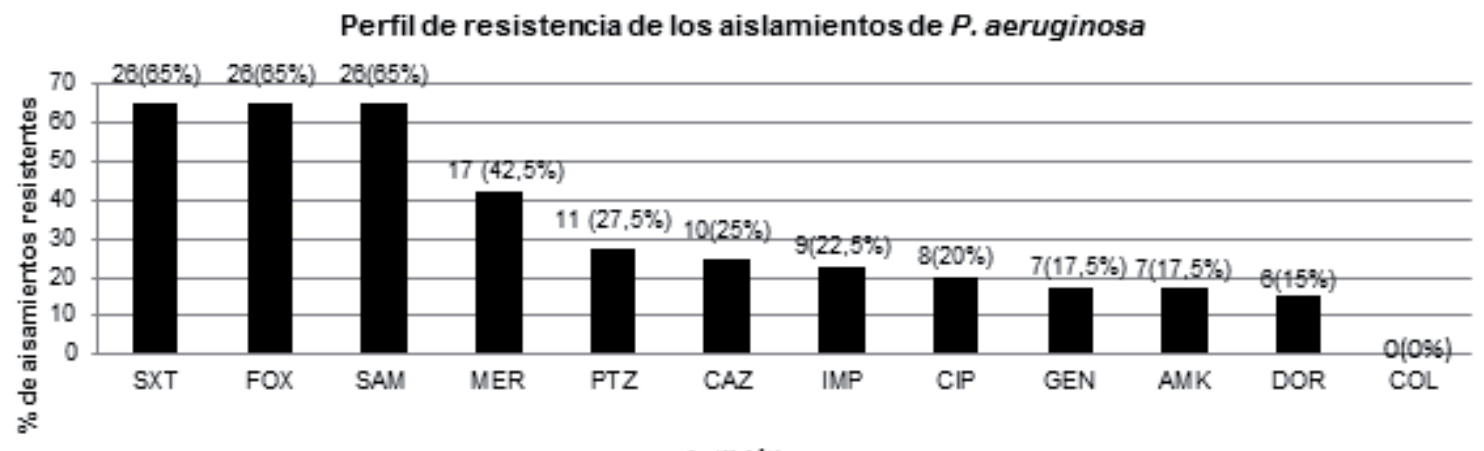

Antibiótico

Figura 1. Perfil de resistencia de los aislamientos de P. aeruginosa; STX: trimetoprim-sulfametoxazol, FOX: cefoxitin, CRO: ceftriazona, SAM: ampicilina/ sulbactam, MER: meropenem, PTZ: piperacilina tazobactam, FEP: cefepime, CAZ: ceftazidima, IMP: imipenem, GEN: gentamicina, AMK: amikacina, DOR: doripenem, COL: colistina.

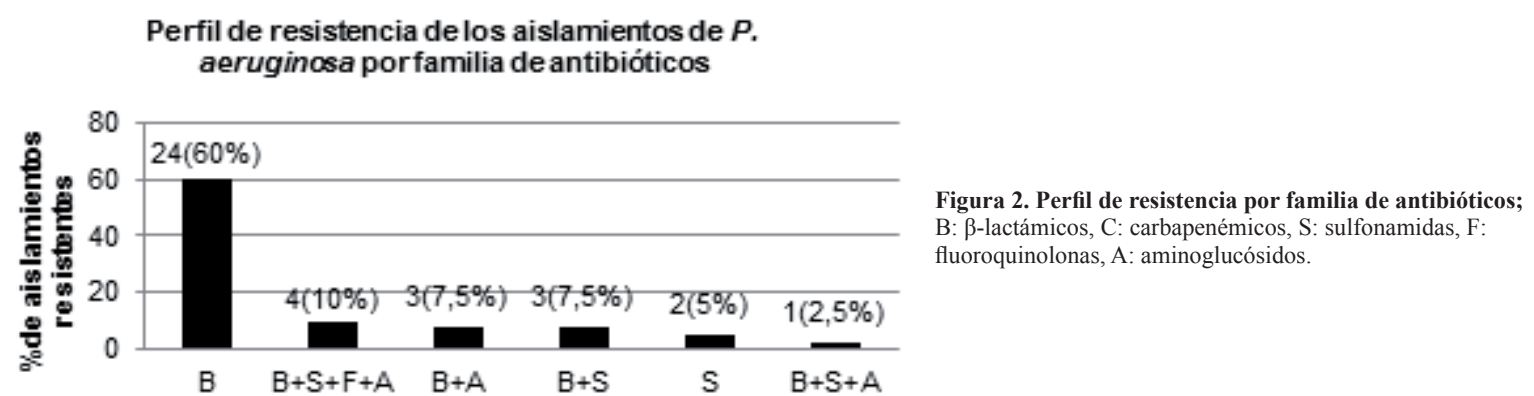

Familias de antibióticos 


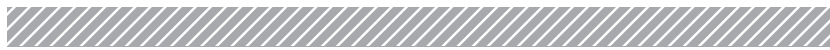

\section{Caracterización molecular de genes de resistencia}

A todos los aislamientos se les determinó por medio de PCR la presencia de genes de resistencia $\beta$-lactámicos, quinolonas, aminoglucósidos y sulfonamidas. En el caso de los antibióticos $\beta$-lactámicos se amplificaron $\beta$-lactamasas de espectro extendido tipo blaTEM, en $8(20,0 \%)$ de los aislamientos, seguida de blaSHV $7(17.5 \%)$ y blaCTX-M 3(7.5\%), carbapenemasas blaKPC-2 y blaVIM en $4(10,0 \%)$ y $3(7.5 \%)$ aislamientos respectivamente. En ninguno de los aislamientos se identificó ampC plasmídico (CIT,FOX,DHA,EBC, ACC,MOX), carbapenemasas tipo blaOXA48, blaGES, blaNDM, blaIMP. Respecto a la resistencia a quinolonas no se detectó la presencia de los genes qnrA, qnrB, qnrS, mcbg y aac6'ib-cr. En sólo 1(2,5\%) aislamiento se encontró el gen aac6'ib asociado con resistencia a aminoglucósidos.

\section{Correlación de resistencia fenotípica y genotípica a carbapenémicos.}

Se identificó que 32(80,0\%) aislamientos presentaron un fenotipo de resistencia a carbapenémicos de los cuales $17(42,5 \%)$ eran resistentes a meropenem, $9(22,5 \%)$ a imipenem y $6(15,0 \%)$ a doripenem. De los aislamientos resistentes a carbapenémicos $4(23,5 \%)$ presentaban la carbapenemasa blaKPC-2 y presentan un fenotipo de resistencia a IMI, MER, DOR y 3(17,6\%) aislamientos que presentan blaVIM solo presentaron resistencia a IMI.

\section{Relación genética}

Los análisis de PFGE mostraron que los 40 aislamientos analizados se agruparon en 28 pulsotipos (comportamiento policlonal), indicando una gran variabilidad genética teniendo en cuenta un porcentaje de similitud de 80. Sin embargo los que presentaron resistencia a carbapenémicos por la presencian de blaKPC-2 pertenecen a un mismo pulsotipo asociado al ST235 y los que presentan blaVIM están asociados al ST111. Estos clones se identificaron en las instituciones de Pereira y Bogotá respectivamente.

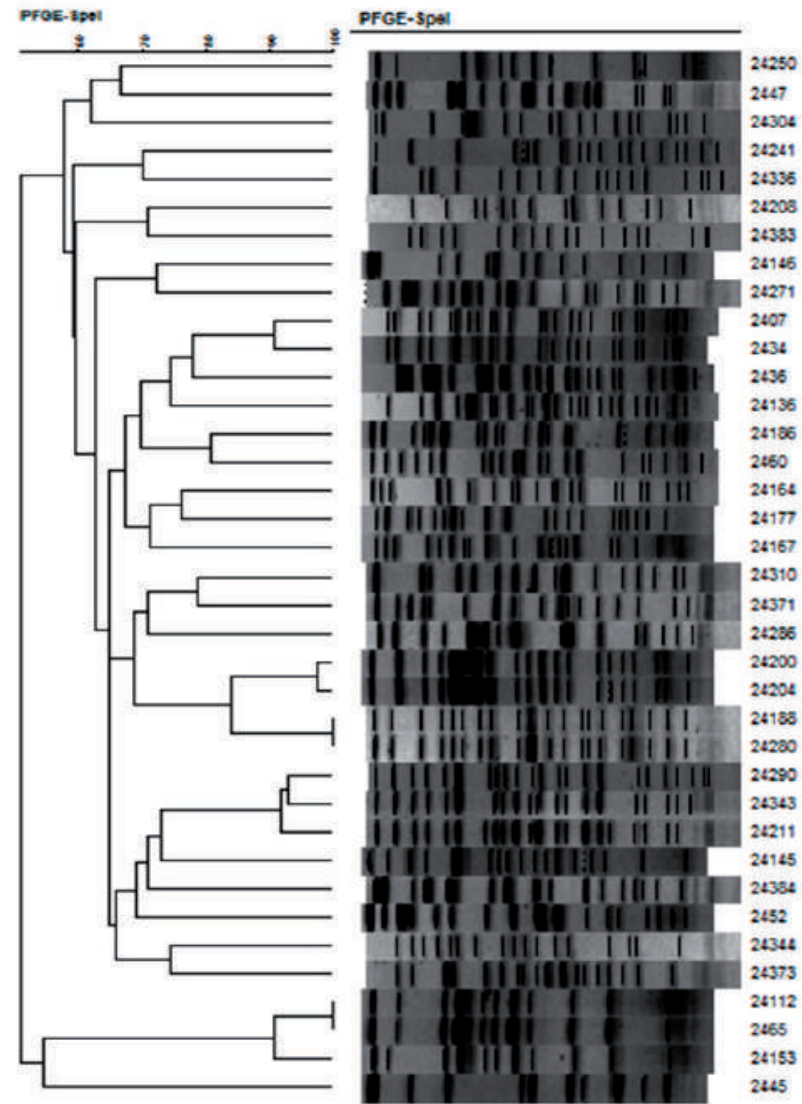

Figura 3. Relación genética de los aislamientos de Pseudomonas aeruginosa.

\section{DISCUSIÓN}

En Colombia P.aeruginosa ocupa el tercer lugar de los patógenos de mayor impacto clínicos en UCI. En los últimos años ha incrementado la resistencia a los antibióticos de última generación como carbapenémicos. Esto se debe a la adquisición de determinantes de resistencia como KPC-2 y VIM [1]. Estos mecanismos de resistencia se han reportado en las instituciones hospitalarias colombianas desde el año 2012 observando un notorio incremento de la frecuencia de circulación en los últimos años $[1,2]$. Dicho comportamiento también fue observado en las UCI analizadas en el presente estudio lo cual presenta un mayor impacto debido a la gravedad de los pacientes que se encuentras en las UCIs, lo que se podría ver reflejado en el incremento de la morbimortalidad y en los costos clínicos asociados a la atención en salud. Sumado a esto se observó una alta frecuencia de infecciones complicadas como bacteremias y neumonías que podrían desencadenar en síndromes sistémicos o incluso la muerte.

Estas infecciones están siendo generado por varios genotipos de estos patógenos, lo cual indica que no hay clon predominante, En los aislamientos resistentes a 
antibióticos carbapenémicos hay un predominio de carbapenemasas tipo KPC-2 en la institución de la ciudad de Pereira y VIM en la de la Bogotá. Estos están relacionados con los clones ST235 y ST111 que se han descrito previamente en Colombia y en varios países del mundo [3]. Los resultados indican que los elementos genéticos móviles que transportan estos genes de resistencia han sido adquiridos por diferentes linajes genéticos lo cual podría estar facilitando su rápida diseminación más la selección ejercida para el uso indiscriminado de antibióticos de última opción $[4,5]$.

\section{CONCLUSIONES}

Las infecciones por Pseudomonas aeruginosa en la población de estudio presentan un comportamiento policlonal, donde no se evidencia un clon predominante. La resistencia a carbapenémicos se encuentra en un $27.5 \%$ la cual se debe mayoritariamente a la producción de carbapenemasas como VIM Y KPC-2. Sin embargo en algunos aislamientos resistentes a carbapenémicos no se identificó la presencia de las carbapenemasas predominantes, lo que sugiere que la resistencia en estos microorganismos esta mediada por otro tipo de mecanismos como bombas de flujo, perdida de porinas u otras carbapenemasas poco frecuentes.

\section{REFERENCIAS BIBLIOGRÁFICAS}

1. Vanegas JM, Cienfuegos AV, Ocampo AM, Lopez L, del Corral H, Roncancio G, et al. Similar frequencies of Pseudomonas aeruginosa isolates producing KPC and VIM carbapenemases in diverse genetic clones at tertiary-care hospitals in Mede1lin, Colombia. Journal of clinical microbiology. 2014;52:3978-86.

2. Martinez E, Perez JE, Buelvas F, Tovar C, Vanegas $\mathrm{N}$, Stokes HW. Establishment and multi drug resistance evolution of ST235 Pseudomonas aeruginosa strains in the intensive care unit of a Colombian hospital. Research in microbiology. 2014;165:8526.

3. Oliver A, Mulet X, Lopez-Causape C, Juan C. The increasing threat of Pseudomonas aeruginosa high-risk clones. Drug resistance updates : reviews and commentaries in antimicrobial and anticancer chemotherapy. 2015.

4. Correa A, Del Campo R, Perenguez M, Blanco VM, Rodriguez-Banos M, Perez F, et al. Dissemination of high-risk clones of extensively drug-resistant Pseudomonas aeruginosa in colombia. Antimicro- bial agents and chemotherapy. 2015;59:2421-5.

5. Shimizu W, Kayama S, Kouda S, Ogura Y, Kobayashi K, Shigemoto N, et al. Persistence and epidemic propagation of a Pseudomonas aeruginosa sequence type 235 clone harboring an IS26 composite transposon carrying the blaIMP-1 integron in Hiroshima, Japan, 2005 to 2012. Antimicrobial agents and chemotherapy. 2015;59:2678-87.

Fecha de recepción: 22/09/15

Fecha de aprobación: 07/10/15

Correspondencia: Abril Riaño Deisy Julieth

Email: deisyapril@gmail.com 